\title{
Computation of Odd Geo-Domination Number of a Graph
}

\author{
K. Karthika, A. Anto Kinsley
}

\begin{abstract}
A geodominating set $S \subseteq V$ of a graph $G$ is said to be an odd geo-dominating set if for every vertex $v \in V-S$, $v \in V-S, N(v) \cap S \neq \emptyset$ and $|N(v) \cap S| \equiv 1(\bmod 2)$ The minimum cardinality of the odd geo-dominating set is called odd geo-domination number denoted by $g_{\text {odd }}(G)$. The odd geo-dominating set with cardinality $g_{o d d}(G)$ is called $g_{0 d d}-$ set of $G$. We develop an algorithm to compute an odd geo-domination number of graphs and for some families of graphs.
\end{abstract}

Keywords: geodesic, geodominating set, geodomination number, odd -geodomination number, graph algorithm

\section{INTRODUCTION}

There are interesting applications of geodetic number concepts [3] to the problem of designing the route for a shuttle and network. The different other areas that apply geodetic number concepts are telephone switching centers, facility location, distributed computing, image and video editing, neural networks and data mining. The geodetic number of a graph was introduced in [3, 8] and further studied in [5, 6]. In [6], geodetic sets and geodetic number were referred to as geodominating sets and geodomination number and this terminology is used throughout this paper. Paper [10] motivates us to develop the new definition odd geo-domination number [2]. In this paper we have developed an algorithm to find odd geo-domination number of a graph. Basic tools to develop these algorithms were developed in [1].

\section{II.PRELIMINARIES}

Definition 2.1: [2] A geodominating set $S \subseteq V$ of a graph $G$ is said to be an odd geo-dominating set if for every vertex $v \in V-S, N(v) \cap S \neq \emptyset$ and $|N(v) \cap S| \equiv 1(\bmod 2)$. The minimum cardinality of the odd geo-dominating set is called odd geo-domination number denoted by $g_{\text {odd }}(G)$. The odd geo-dominating set with cardinality $g_{\text {odd }}(G)$ is called godd - set of $G$.

Manuscript received on December 26, 2020.

Revised Manuscript received on January 06, 2021.

Manuscript published on February 28, 2021.

* Correspondence Author

K. Karthika*, Research Scholar, Department of Mathematics, Reg No. 11794, St. Xavier's (Autonomous) College, Palayamkottai, Tirunelveli . Email: karthika2490@gmail.com

A. Anto Kinsley, Department of Mathematics, St. Xavier's (Autonomous) College, Palayamkottai, Tirunelveli. Email: antokinsley@yahoo.com

(C) The Authors. Published by Blue Eyes Intelligence Engineering and Sciences Publication (BEIESP). This is an open access article under the CC BY-NC-ND license (http://creativecommons.org/licenses/by-nc-nd/4.0/)
Definition 2.2: [1] Characterize each closed interval as a $n$-tuple. Each place of $n$-tuple can be represented by a binary 1 or 0 . Call this $n$-tuple as a link vector. Denote $L V(I)=I^{\prime}$. Put 1 if the vertex belongs to the closed interval otherwise 0 . If all the co-ordinate of the link vector are equal to 1 then it is called as full. Denote $I[(1)]$.

Theorem 2.3: [2] For any connected graph $G$ $2 \leq g(G) \leq g_{o d d}(G) \leq n$.

Algorithm 2.4

Algorithm: Geodominating set confirmation

Procedure geodetic $[S]$

Input: A graph $G=(V, E), V=\left\{v_{1}, v_{2}, \ldots, v_{n}\right\}$, a subset $S=$ $\left\{v_{1}, v_{2}, \ldots, v_{k}\right\}$

Output: $S$ is geodominating set or not.

begin

Step 1: Find all the 2-subsets $S_{2}$ of $S$

\{There are $\left(\begin{array}{l}k \\ 2\end{array}\right)$ number of subsets $S_{2}$ of $S$ \}

Step 2: Take $L \leftarrow(0)$

Step 3: for $i=1$ to $\left(\begin{array}{l}k \\ 2\end{array}\right)$

begin

Closed- interval $I_{i}\left[S_{2}\right]$

Link vector $I_{i}^{\prime}\left[S_{2}\right]$

$L=L \vee I_{i}^{\prime}\left[S_{2}\right]$

end

Step 4: If $L$ is full, then Print: "The given set $S$ is geodetic", Stop.

Otherwise, Print: "The given set $S$ is not geodetic", End

In this algorithm, step 2 will work in $\frac{k(k-1)}{2}$ times.

Next part of step 2 is the algorithm Closed-interval $I_{i}\left[S_{2}\right]$ and Link vector $I_{i}^{y}\left[S_{2}\right]$ and hence this part will work with $2 m+n$ verifications. Total cost of time is $O\left(k^{2}(m+n)\right.$, where $k$ is the cardinality of the given vertex subset and $m$ is the number of edges in $G$. But in this step the given vertex acts as a root and all other vertices are approached through a spanning tree. Therefore there are $n+(n-1)$ verifications needed, since $m=n-1$ for a tree. That is, $O\left(n^{2}(2 n-1)\right)$, that is $O\left(n^{3}\right)$. Thus this algorithm requires $O\left(n^{3}\right)$ cost of time, finally we develop the main algorithm to find the minimum geodetic set of a graph $G$.

\section{MAIN RESULTS}

In this section, we define the algorithms to compute odd geo-domination in a graph $G$

Algorithm 3.1:

Algorithm: Set $N\left(v_{i}\right)$

\section{Published By:}

Blue Eyes Intelligence Engineering and Sciences Publication

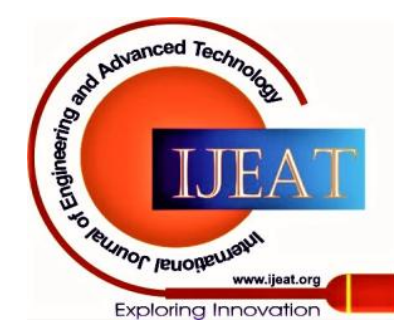




\section{Computation of Odd Geo-Domination Number of a Graph}

To find $N\left(v_{i}\right)$ for a vertex $v_{i}$ :

Input: A graph $G=(V, E)$, adjacency matrix $\{a[i, j]\}$ and the vertex $v_{i}$

Output: $N\left(v_{i}\right)$

Step 1: Take $N\left(v_{i}\right)=\emptyset$

Step 2: for $j=1$ to $n$ do begin

$$
\text { if } a[i, j]=1 \text {, then } N\left(v_{i}\right)=N\left(v_{i}\right) \cup\left\{v_{j}\right\}
$$

end.

Here the algorithm takes n verifications. That is, it works $O(n)$ cost of times.

\section{Algorithm 3.2 \\ Algorithm: Compute $\left|\boldsymbol{N}\left(v_{i}\right) \cap \mathrm{S}\right|$}

To find $\left|N\left(v_{i}\right) \cap \mathrm{S}\right| ; v_{i} \in V-S$

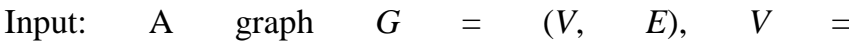
$\left\{v_{1}, v_{2}, \ldots, v_{n}\right\}, v_{i} \in V-S \times S=\left\{v_{1}, v_{2}, \ldots, v_{k}\right\}$ and degree sequence $\left\{d\left(v_{i}\right)\right\}$.

Output: $\left|N\left(v_{i}\right) \cap \mathrm{S}\right|$

Step 1: Set $N\left(v_{i}\right)$

Step 2: Take count $=0$

Step 3: for $j=1$ to $d\left(v_{i}\right)$

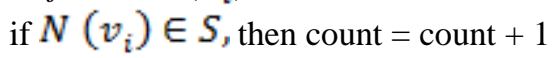

Step 4: Print $\left|N\left(v_{i}\right) \cap \mathrm{S}\right|=$ count

By step 1, it takes $n$ verifications. By Step 3, it works in deg $\left(v_{\mathrm{i}}\right)$ number of times to check whether the neighborhood of $v_{\mathrm{i}}$ belongs to $\mathrm{S}$ or not. That is, totally it works in $\operatorname{deg}\left(v_{\mathrm{i}}\right)=k$ (say) times. Thus it requires $O(n+k)$ cost of time.

\section{Algorithm 3.3}

\section{Procedure odd geo-dominating set [S]}

Input: A graph $G=(V, E), V=\left\{v_{1}, v_{2}, \ldots, v_{n}\right\}, S=$ $\left\{v_{1}, v_{2}, \ldots, v_{k}\right\}$ and $V-S=\left\{v_{k+1}, v_{k+2}, \ldots, v_{n}\right\}$

Output: $S$ is odd geo-dominating or not.

Step 1: procedure geodetic $[S]$;

Step 2: if $S$ is geodetic, then

for $i=k+1$ to $n$

procedure $\left[\left|N\left(v_{i}\right) \cap \mathrm{S}\right|\right]$

Step 3: if $\left|N\left(v_{i}\right) \cap \mathrm{S}\right| \bmod 2 \equiv 0$, then $S$ is not an odd geo-dominating set.

Stop.

Otherwise $S$ is an odd geo-dominating set.

In step 1, we call Producer geodetic [S], it requires $O\left(n^{3}\right)$ cost of time. Next part of the algorithm is for loop which works for $n-k$ times. In step 2 , the Producer $\left|N\left(v_{i}\right) \cap S\right|_{x}$ it it requires $O(n+k)$ cost of time. Thus it requires $O\left(n^{5}\right)$ cost of time.

Algorithm 3.4

Algorithm to find minimum odd geo-dominating set:

Input: A graph $G=(V, E)$ with its vertex set $V=$ $\left\{v_{1}, v_{2}, \ldots, v_{n}\right\}$.

Output: $g_{\text {odd }}$ set of $G$.

Step 1: Take $k \leftarrow 2$

Step 2: Take all the subsets $S_{j}\left(1 \leq j \leq\left(\begin{array}{l}n \\ k\end{array}\right)\right)$ of $V$ with $k$ vertices.

Step 3: for $j=1$ to $\left(\begin{array}{l}n \\ k\end{array}\right)$

begin

procedure odd geo-dominating set $\left[S_{j}\right]$ if yes then stop and print $S_{j}$ is a $g_{\text {odd }}$ - set of $G$.

end

Step 4: Otherwise take $k=k+1$ and return to step 2 .

End.

In this algorithm, by the for loop it takes all the $2^{n}$ subsets and each time it calls the procedure Odd Geo-dominating [S], it's complexity is $O\left(n^{5}\right)$. Hence this algorithm is $N P$-complete.

\section{CONCLUSION}

Algorithms to compute odd geo-domination number of a graph is discussed in this paper. In further research we will try to reduce the complexity of minimum odd geo-domination number.

\section{REFERENCES}

1. A. Anto Kinsley and K. Karthika, 2016, "Algorithmic Aspects of Geodetic sets in Graphs", International Journal of Applied Science and Mathematics, Volume 3-Issue 01, ISSN (Online): 2394-2894.

2. A. Anto Kinsley and K. Karthika, 2020, “Odd Geo-dominating Number of a Graph", Science, Technology and Development Journal, Volume IX, Issue XII, 442-447, ISSN: 0950-0707.

3. F.Buckley and F. Harary, Distance in Graphs, Addition - Wesley, Redwood City, CA, 1990.

4. Byung Kee Kim, The Geodetic number of a graph, J. Appl. Math. \& Computing Volume 16 (2004), No. 1-2, pp. 525-532.

5. G. Chartrand, F. Harary and P. Zang, On the geodetic number of a graph, Networks. 39(2002) 1-6.

6. G. Chartrand, F. Harary, H. Sart, and P. Zang, Geodomination in graphs, Bull. Inst. Appl. 31(2001), 51-59.

7. G. Chartrand, P. Zang and Kalamazoo, Extreme Geodesic Graphs, Czechoslovak Mathematical Journal, 52(127) (2002). 771 - 780.

8. F. Harary, Graph Theory, Addition - Wesley, 1969.

9. F. Harary, E. Loukakis, C. Tsourous, The geodetic number of a graph, Math. Comput. Modeling, 17(11)(1993), 87-95.

10. Yair Caro, William F. Klostermeyer, The odd domination number of a graph, Journal of Combinatorial and Combinatorial Computing, January 2003.

\section{AUTHORS PROFILE}

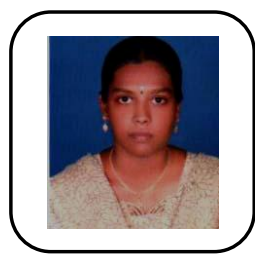

K. Karthika M.Sc., M.Phil. Research scholar in Department of Mathematics in St.Xavier's College (Autonomous), Palayamkottai. Area of Specialization is graph theory. Published 4 papers in National and International Journals. Presented 2 papers in two National Conferences.

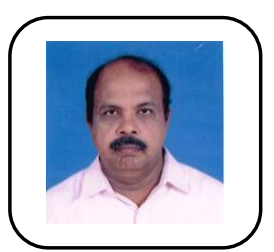

Dr. A. Anto Kinsley M.Sc., M. Phil., M. Tech., Ph.D. Associate Professor in Mathematics in St. Xavier's College (Autonomous), Palayamkottai. He has 30 years teaching experience. He published 28 research articles in National and International Journals and he completed two UGC minor research projects. His area of specializations are Graph Algorithms, Flow Networks. Distance in Graphs, Central structures in Graphs. Domination and Distance domination in graphs, Star Graphs, Convexity in Graphs, DNA computing.
Published By:

Blue Eyes Intelligence Engineering and Sciences Publication (C) Copyright: All rights reserved.

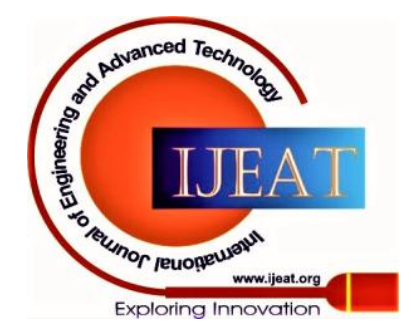

